\title{
La sociología urbana francesa y la evolución de las politicas urbanas de los años 1960 a los años 1980: ¿porosidades, impermeabilidades $o$ afinidades electivas?
}

\author{
French urban sociology and urban policies development from \\ the 1960s to the 1980s: porosity, impermeability or elective \\ affinity?
}

\author{
Grégory Busquet \\ Université Paris Ouest Nanterre \\ gbusquet@u-paris10.fr (FRANCIA) \\ [Traducción de Emilio Martínez Gutiérrez. UCM]
}

Recibido: 22.11 .2012

Aceptado: 01.042013

\section{RESUME}

La sociologie urbaine française s'est développée conjointement aux politiques urbaines françaises depuis les années 1960. Lorsque ces politiques appréhendaient le fait urbain dans sa globalité, la sociologie faisait de même. Quand, à la fin des années 1970, la sociologie se recentre sur l'échelle local, l'urbanisme en fait également de même. C'est par une nébuleuse d'acteurs allant de la deuxième gauche aux sociologues marxistes en passant par les mouvements sociaux urbains et par les idées qu'ils véhiculent comme celle, relativement nouvelle, de la participation, ou celles plus ancienne de la mixité résidentielle (versus la ségrégation) ou du spatialisme, que se réalise cette double évolution. Ce faisant, on assiste à une dépolitisation progressive de la discipline et, plus largement de la question urbaine et de la question sociale, ce qui dénote un changement dans les usages sociaux de la sociologie urbaine et dans le rapport des sociologues au pouvoir. Si les idées guidant les politiques urbaines et la tentative d'appréhension et de compréhension des sociétés urbaines restent les mêmes, seule la question de l'échelle varie et la vision politique se tarit. 


\title{
RESUMEN
}

La sociología urbana francesa se desarrolló conjuntamente a las políticas urbanas francesas desde los años 1960. Cuando estas políticas concebían el hecho urbano en su carácter global, la sociología hacía lo mismo. Cuando a finales de los años 1970 la sociología se orienta de nuevo sobre la escala local, el urbanismo hace otro tanto. Esta doble evolución se realiza por una amalgama de actores que va de la «segunda izquierda» a los sociólogos marxistas, pasando por los movimientos sociales urbanos y por las ideas que estos transportan, como la relativamente nueva de participación o las más antiguas del carácter mixto del alojamiento (versus la segregación) o del «espacialismo». De este modo, asistimos a una despolitización progresiva de la disciplina y más ampliamente de la cuestión urbana y de la cuestión social, lo que denota un cambio en los usos sociales de la sociología urbana y en la relación de los sociólogos con el poder. Si las ideas que guían las políticas urbanas, la manera de ver y de entender las sociedades urbanas, permanecen iguales, sólo varía la cuestión de la escala y la visión política se diluye.

\section{PALABRAS CLAVE}

Sociología urbana/ Planificación urbana/Políticas urbanas/ Movimientos sociales.

\begin{abstract}
French urban sociology developed in conjunction with French urban policy since the 1960s. By the time these policies were approaching the urban as a whole, sociology was doing the same. In the late 1970s, when sociology was focusing on the local level, town planning was evolving in a similar manner. A heterogeneous set of actors contributed to this evolution: from the «second left wing» to Marxist sociologists, including urban social movements and the ideas they conveyed, such as the relatively new «participatory democracy», or the older - «mixed residential areas» (as opposed to segregation) or «spatial determinism». In doing so, a gradual depoliticization of the discipline occurred and, more broadly, of the urban and of the social issues. This indicates a shift in the social uses of urban sociology and in sociologists' relation to power. While the ideas guiding urban policies and the attempt to apprehend and understand urban societies in reality remained the same, what varied was a matter of scale, as well as of political vision that ran out.
\end{abstract}

\section{KEYWORDS}

Urban Sociology / Town Planning/ Urban Policies / Social Movements.

EMPIRIA. Revista de Metodología de Ciencias Sociales. N. 27, enero-abril, 2014, pp. 121-136. ISSN: 1139-5737 DOI 10.5944/empiria.27.10865 


\section{INTRODUCCIÓN}

A veces resulta conveniente que una ciencia social se vuelva hacia su pasado con el fin de evaluar cuáles han podido ser sus efectos sobre las decisiones tomadas y sobre el mundo social. En ese sentido es legítimo plantearse la cuestión de los usos sociales de la sociología urbana y cuál es su lugar en el campo de la intervención pública, en particular en las políticas urbanas. La socióloga Sylvie Tissot, por ejemplo, ha mostrado el papel de la sociología de Touraine en la implantación de la «política de la ciudad» ${ }^{1}$ y en la elaboración de las categorías de pensamiento de la acción pública sobre la periferia francesa, en términos de exclusión, de lucha contra la formación de guetos, etc. (Tissot 2007). Nuestro propósito es remontarnos más allá y aspirar a una perspectiva de mayor alcance temporal, examinando cómo la sociología urbana francesa ha evolucionado desde 1960, acompañando al cambio de las políticas urbanas y aportando una mirada nueva sobre la ciudad y el fenómeno urbano.

Sería muy presuntuoso o aventurado afirmar que los sociólogos han influido de un modo general sobre la acción pública o que han podido provocar en ella giros radicales, incluso cuando se han constatado ciertas «recuperaciones» posteriores de sus tesis por parte de los políticos (Garnier y Goldschmidt 1978). Por eso preferimos hablar aquí de acompañamiento, pues los sociólogos y quienes toman las decisiones hacen progresar la sociología y la acción pública conjuntamente, hallando cierto interés común en su alianza — lo que motiva que vayan a veces en el mismo sentido, pero no siempre. Esto nos lleva a indagar en las eventuales interacciones entre los actores e ideas de ambas esferas, cuyos encuentros habrían podido comportar - en lo ideal - mudanzas en la forma de concebir el fenómeno urbano, en la sociología urbana francesa e incluso, por qué no, en la acción pública. Dicho de otro modo, no se trata de analizar cómo la acción pública determina a la sociología o viceversa - lo que sería algo ingenuo por otra parte- sino más simplemente determinar lo que una y otra se deben mutuamente.

Nuestro interés versa sobre lo que se podría designar como una politización de la cuestión urbana, que marca la institucionalización académica de la sociología urbana y que se efectúa conjuntamente a una «urbanización» del pensamiento político (del discurso político para ser más preciso) por diferentes medios, y que concurre, como veremos, en la territorialización de la cuestión social (Busquets 2007). Estos nos llevará a su vez a explorar las aproximaciones teóricas —o más bien «ideológicas»— de las políticas públicas y de la sociología francesa desde hace varias décadas.

A partir de ese hilo conductor entre los actores y sus ideologías es posible indagar en los vínculos entre el pensamiento sociológico y la acción pública

1 Por «política de la ciudad» debemos entender una política interministerial llevada a cabo en Francia en los años 1980 con el fin de resolver los problemas urbanísticos y socioeconómicos de los barrios periféricos formados por grandes polígonos de viviendas sociales («grands ensembles») construidos en la década de 1960, dirigida particularmente a aquellos afectados por el paro, la anomia, la delincuencia y la degradación física.

EMPIRIA. Revista de Metodología de Ciencias Sociales. N. 27, enero-abril, 2014, pp. 121-136. ISSN: 1139-5737 DOI 10.5944/empiria.27.10865 
territorializada, en su evolución, considerando algunos factores externos pero sólidamente ligados —en la institucionalización de la sociología urbana francesa y en la marcha de la acción pública-, a saber, los movimientos sociales y la crítica de las políticas públicas.

Pero antes de explorar los posibles vínculos entre los medios académico y político es preciso considerar algunos elementos que marcan la institucionalización de la sociología urbana francesa y la progresión de las políticas urbanas. Abordaremos a continuación algunos temas y mencionaremos algunos actores clave que han hecho cambiar simultáneamente la intervención sobre lo urbano y el pensamiento sobre la ciudad. Por último, intentaremos determinar cómo ha evolucionado recientemente, y en términos ideológicos, la relación entre la sociología urbana, la acción pública y el poder, pues de lo que tratamos aquí no es sino de las articulaciones y aproximaciones entre la sociología y el poder. Si en Francia la sociología urbana se ha pretendido insistentemente la «conciencia crítica» del planificador (urbanista, arquitecto, autoridad...), lo cierto es que a menudo también ha avalado y proporcionado los útiles para la legitimación de su acción, incluso a su pesar.

\section{INSTITUCIONALIZACIÓN DE LA SOCIOLOGÍA URBANA FRANCESA Y POLÍTICAS URBANAS}

Tras la reconstrucción de viviendas, redes e infraestructuras que siguió a la Segunda Guerra mundial, hacia mediados de los años 1950 tuvo lugar una urbanización masiva y acelerada bajo iniciativa de los poderes públicos que vino acompañada en Francia del desarrollo de la investigación urbana (1967), al amparo del nuevo Ministère de l'équipement de Edgar Pisani creado en 1966. En ese contexto se institucionalizó la sociología urbana en Francia, aun cuando la ciudad y lo urbano fueran objetos privilegiados de las ciencias sociales desde los años 1950.

El urbanismo de los «Treinta gloriosos» — periodo de crecimiento económico, auge demográfico y desarrollo de la sociedad de consumo - vino marcado por la construcción de grandes conjuntos de viviendas en la periferia de las grandes aglomeraciones, destinadas a alojar a la mano de obra rápidamente y al menor coste (Bachmann y Le Guennec 1995). Esos grandes polígonos tenían como objetivo, al menos inicialmente, reunir a las clases media y obrera, remitiéndose a una ideología civilizadora ligada a la idea de una mezcla social. Pero los problemas de vecindad y de sociabilidad se dejaron sentir muy pronto como resultado de la proximidad entre grupos sociales heterogéneos (Chamboredon y Lemaire 1970). Tras la salida de las capas medias, el Estado francés decidió tomar las riendas desde finales de los años 1970 de este patrimonio cuya degradación física y evolución social llevaba a numerosos expertos y comentaristas a hablar del «malestar» o del «mal de las periferias» y progresivamente de «guetos» (Maurin 2004). Es así como cobró forma la denominada «política de la

EMPIRIA. Revista de Metodología de Ciencias Sociales. N. ${ }^{2}$ 27, enero-abril, 2014, pp. 121-136. ISSN: 1139-5737 DOI 10.5944/empiria.27.10865 
ciudad» destinada a enmendar social, económica y urbanísticamente la deriva de tales barrios en enclaves.

Visto en perspectiva, ese urbanismo de posguerra se caracterizó por un fuerte voluntarismo político y un intervencionismo estatal que retomaba, por mediación de los diferentes ministros de la Reconstrucción, las ideas de la Carta de Atenas, teñidas de una ideología modernista y civilizadora de transformación social por la disposición arquitectónica y la voluntad política. Los arquitectos de los grandes conjuntos navegaron en ese contexto.

Paralelamente, la sociología urbana francesa, surgida del estallido de la disciplina en diferentes campos y cuyos representantes no eran todavía numerosos -Henri Lefebvre, Paul-Henry Chombart de Lauwe, Raymond Ledrut y sus discípulos, esencialmente- se desarrollaría en la segunda mitad de los años 1960, entre otras cosas, en torno a la crítica de ese urbanismo, y eso incluso cuando, durante un tiempo, algunos pensadores de la sociedad urbana creyeron ver en esos grandes conjuntos habitacionales el sustrato de una nueva sociedad y del cambio social (Chombart de Lauwe, 1963) ${ }^{2}$.

Pero más allá, esta sociología incipiente comenzaba a plantearse las cuestiones de su relación con la toma y los sujetos de las decisiones. Así, durante el coloquio de Royaumont titulado «sociología y urbanismo», que la consagra en 1968, los temas abordados se hicieron claramente eco de las preocupaciones del momento pero denotaban asimismo el posicionamiento político de la disciplina in fieri: se discutían los temas clásicos (o los que lo serían con el tiempo) de las segregaciones urbanas, la mezcla social, la información y participación ciudadana en las decisiones, pero también el propio engarce de los sociólogos en el requerimiento público, mediante cuestiones tales como la integración del sociólogo en calidad de «sujeto de síntesis» o de simple «conciencia crítica» en el equipo pluridisciplinar del urbanismo. Se examinaba también «hasta qué punto la investigación podía depender de la encomienda» o «cómo formar parte de la decisión» sin ser únicamente un ámbito de apreciación o de consejo (Foundation Royaumont 1970). Conviene recordar al respecto que durante los años 1960 los sociólogos fueron menos solicitados y contribuyeron en menor medida que sus homólogos de otras ciencias sociales — como, por ejemplo, los geógrafos-a las políticas de urbanización francesas ${ }^{3}$.

Sea como fuere, a finales de la década de 1960, los sociólogos urbanos se reclaman mayoritariamente marxistas y aunque fuertemente dependientes de la demanda pública, se aplican en buena medida a introducir la crítica en sus estudios e investigaciones. Así hacen los diferentes laboratorios de sociología urbana, se trate del Centre de sociologie urbaine (CSU) fundado en 1954 por Paul-Henry Chombart de Lauwe; del Centre d'étude des mouvements sociaux (CEMS) de

2 Véase a este respecto la crítica de Jean-Claude CHAMBOREDON y Madeleine LEMAIRE en « Proximité spatiale et distance sociale : les grands ensembles et leur peuplement », Revue française de sociologie, $\mathrm{n}^{\circ}$ XI-1, Paris, 1970.

3 Por ejemplo, el esquema de la aglomeración de París, la política de las ciudades nuevas, las «metrópolis de equilibrio» o más generalmente la ordenación del territorio en su conjunto, en los que los geógrafos han influido ampliamente y participado ocasionalmente.

EMPIRIA. Revista de Metodología de Ciencias Sociales. N. . 27, enero-abril, 2014, pp. 121-136. ISSN: 1139-5737 DOI 10.5944/empiria.27.10865 
Alain Touraine; del Centre d'études, de recherche et de formation institutionnelles (CERFI) foucaltiano, o por supuesto del Institut de sociologie urbaine (ISU) de Henri Lefebvre. En todos ellos se advierte, unas veces por la renovación generacional y otras desde su misma creación, cómo la crítica antiautoritaria de la planificación o la crítica marxista (bajo distintas formas) de las políticas urbanas o del urbanismo se convierte en el eje esencial de sus estudios y teorías.

La sociología urbana experimentó desde los sesenta y setenta del pasado siglo una clara politización influida por el clima contestatario y la coyuntura sociopolítica del periodo (Lassave 1997; Garnier 2008). Esa sociología —o más bien «esos» sociólogos - se desplegaron en «un espacio tripolar», por retomar la expresión de Michel Amiot (1986): los partidos políticos («del partido comunista a la extrema izquierda»), «los organismos académicos» (Universidad, CNRS) y el Estado.

Desde el punto de vista del contenido, todo el pensamiento urbano crítico analiza - con aproximaciones diversificadas - la ciudad y lo urbano en términos de alienación, de lucha de clases, de divisiones, de dominaciones y de producción-consumo. La ordenación, fuera estatal o «liberal», se percibía y denunciaba como un instrumento de clase, de la dominación, de la represión; y la ciudad, como una materialización espacial del poder (de la burguesía, del Estado).

En la época, esa sociología urbana en vías de estructuración iba a la búsqueda de un objeto que encontraría inicialmente en la crítica de los modos de producción del espacio así como en el análisis de los movimientos sociales -únicos objetos pertinentes de la sociología urbana, escribía Manuel Castells en la revista Sociologie du travail (1968). A través del urbanismo y las políticas urbanas, el Estado mismo y el modo de producción capitalista son denunciados básicamente mediante la crítica del célebre «capitalismo monopolista de Estado» (Lojkine 1977). Nada de eso era nuevo; la novedad radicaba en que se les criticaba en y por el espacio (su organización, su producción).

\section{LOS ACTORES Y SUS IDEAS: LA SOCIOLOGÍA URBANA MARXISTA, LOS MOVIMIENTOS SOCIALES URBANOS Y LA «SEGUNDA IZQUIERDA»}

La sociología y las políticas urbanas permitieron evidentemente hacer evolucionar la visión sobre la ciudad y la planificación. Pero si hay que establecer y esclarecer sus vínculos es ineludible escudriñar entre los actores participantes: actores con ideas propias, que actúan según sus intereses, motivaciones y sistemas de valores particulares.

La sociología urbana francesa, cuyos estudios y encuestas eran financiados por el Estado, mantenía relaciones de dependencia con los servicios estatales mientras criticaba las políticas urbanas. Para explicar esas relaciones con la acción pública debemos remontarnos a la era gaullista. A los aspectos mayoritariamente «cuantitativos» de ese periodo (atajar la «crisis de la vivienda», construir velozmente numerosas viviendas, las infraestructuras y los equipamientos 
necesarios), los sociólogos críticos opusieron otros argumentos sociales más «cualitativos» relativos a los grandes conjuntos, la vida social degradada y la inadaptación del hábitat, la rigidez del marco de vida, el alejamiento de los centros, las segregaciones socio-espaciales... Esos temas les permitían enjuiciar la planificación urbana proporcionando al tiempo objetos para sus investigaciones: no era sólo una sociología crítica, pues también estaba interesada en los objetos sociológicos, y la crítica de los grandes conjuntos se hacía en nombre de los habitantes, de sus modelos socioculturales, de sus sociabilidades, etc. Además, ante la constatación de la obsolescencia precoz -incluso inadaptación- de ese tipo de hábitat, los poderes públicos invocaron a los nuevos sociólogos de la ciudad y de las «periferias» buscando diagnósticos y soluciones Algunos harían así su carrera y muchos continúan hoy día operando en el mismo sentido.

Debemos asimismo hacer referencia a los movimientos sociales urbanos de los años setenta, que participaban entonces de una «politización de la cuestión urbana», por retomar los términos de M. Castells (1972). Constituyeron la crítica actuada de las políticas urbanas emprendidas en Francia desde décadas atrás. Por supuesto, las luchas urbanas no datan de ese periodo pero las que se extienden a partir de finales de 1960 se caracterizaron también, entre otros factores, por el paso de reivindicaciones «cuantitativas» (relativas al número de viviendas, precio de alquileres...) a pretensiones más «cualitativas» concernientes fundamentalmente a los modos y la calidad de vida, la emancipación política y social, las reivindicaciones de las «minorías» y, por supuesto, la preservación o transformación del famoso «marco de vida», así como las exigencias relativas a una nueva arquitectura. No se trata aquí de ilustrar ni de aplicar tal cual al espacio urbano las tesis de la sociología de los «nuevos movimientos sociales» de Alain Touraine (1970-1980), según la cual el movimiento social pasaría de tipos reivindicativos cuantitativos (salarios, empleos) a tipos cualitativos (modos de vida, igualdad...) (1978). Pero es forzoso constatar que las consignas de las luchas urbanas cambiaron en Francia entre 1960-1970.

Ciertamente la carencia de alojamiento fue corregida (Coing, Topalov 1995). Pero hay que indicar también que esos movimientos sociales urbanos experimentaron en el curso de los acontecimientos una modificación profunda de sus componentes sociológicos: la presencia de las capas medias era cada vez mayor, actuando como agentes movilizadores de las poblaciones implicadas en la lucha. A medida que transcurría el decenio, sin embargo, las reivindicaciones cualitativas formuladas por las clases medias-«los militantes del marco de vida» (Dagnaud \& Mehl, 1981 ; Bidou, 1984 ; Bacqué \& Vermeersch, 2007)-, ligadas a las condiciones de vida en la ciudad, se centraban progresivamente en la defensa de su propio entorno vital. Los movimientos opositores a las operaciones urbanísticas y a los proyectos que les afectaban directamente fueron protagonizados cada vez más por residentes, ribereños y comerciantes del barrio. También aparecieron reivindicaciones de autogestión urbana y del urbanismo participativo que desde los años sesenta promovían en el seno de las ciencias sociales los sociólogos Raymond Ledrut (1968) y sobre todo Henri Lefebvre $(1968,1970)$ en respuesta al urbanismo del periodo gaullista, juzgado tecnocrático y autoritario.

EMPIRIA. Revista de Metodología de Ciencias Sociales. N. . 27, enero-abril, 2014, pp. 121-136. ISSN: 1139-5737 DOI 10.5944/empiria.27.10865 
Dentro del movimiento social, las reivindicaciones sociales y políticas por el «marco de vida» se abrirían más tarde a las críticas hacia el automóvil, la contaminación y diversas molestias urbanas así como a demandas relativas a los espacios verdes: en suma, a la ecología política urbana. Las diferentes asociaciones que se desarrollan en ese sentido reclamaban un entorno de «proximidad», y vinculadas con el movimiento antinuclear contemporáneo, alumbrarían la ecología política en Francia.

La sociología urbana, especialmente el CSU, se interesará de soslayo en esos movimientos sociales y en sus reivindicaciones. Para algunos, como M. Castells, eran la otra vertiente de una lucha de clases que se desplegaría en adelante en el espacio urbano, además de una alternativa potencial a la producción capitalista del espacio (1972, 1974). El CERFI foucaultiano, se implicó en esos movimientos, principalmente durante el conflicto de Alma-Gare en Roubaix, promoviendo la idea de un urbanismo participativo y el contraproyecto de los habitantes. En esa época, los sociólogos urbanos preconizaban de hecho el diálogo atento entre la administración, los planificadores, los habitantes y los usuarios, y la toma en consideración de las necesidades y aspiraciones ciudadanas.

Pero los sociólogos no influyeron únicamente en la esfera de las ciencias de lo urbano ni se involucraron exclusivamente en los movimientos sociales; también dejaron sentir su influencia en ámbitos de la oposición política. Henri Lefebvre, compañero de viaje del Partido Comunista francés (PCF) desde los años 1920 y excluido hacia 1950, se acercó a la «segunda izquierda» autogestionaria y al Partido Socialista Unificado (PSU) hacia 1960 sin llegar a adherirse a él jamás. Como Chombart de Lauwe, era un intelectual próximo a Michel Rocard ${ }^{4}$ y a otros cuadros del partido; asimismo, fue uno de los teóricos de la autogestión (1960-1970), dentro de este movimiento que iba de los medios libertarios a la «segunda izquierda» pasando por el conseillisme.

Claramente inspirados por las teorías lefebvrianas, el PSU, el GAM (Grupos de acción municipal), el sindicato CFDT (Confederación francesa democrática del trabajo) y el ADELS (Asociación para la democracia local y la educación social) —es decir, el conjunto de la «segunda izquierda» de finales de 1960 y de los años 1970 - defendían un nuevo urbanismo, un nuevo modo de producción del espacio no capitalista, y la reinvención de una nueva cotidianidad a recrear, apoyando por completo a los reconocidos movimientos sociales urbanos (de inquilinos, usuarios y después de la ecología política urbana). El célebre «derecho a la ciudad» lefebvriano se convertiría en un tema de campaña en 1968 (Busquets 2007b). De modo general, los partidos políticos penetraron o animaron esos «movimientos sociales urbanos» (el PCF con la Confederación nacional de inquilinos, la «segunda izquierda» y el catolicismo social con la Confederación general de vivienda o incluso «el sindicalismo del marco de vida», etc.).

4 Jefe de filas de la segunda izquierda francesa, desde finales de 1960 a principios de los 70. Más tarde adversario y rival de F. Mitterrand dentro del PS, si bien será Jefe de Gobierno de éste tras su reelección a la Presidencia de la República en 1988.

EMPIRIA. Revista de Metodología de Ciencias Sociales. N. ${ }^{\circ}$ 27, enero-abril, 2014, pp. 121-136. ISSN: 1139-5737 DOI 10.5944/empiria.27.10865 
En 1974 buena parte de los miembros de la «segunda izquierda» vinieron a integrarse en el nuevo partido socialista. Sus ideas pro-localistas, participativas y autogestionarias pasan así al partido e informan ampliamente sus programas políticos (fundamentalmente durante la campaña de las municipales de 1977 y la presidencial de 1981). Esas ideas influyeron en la descentralización y sirvieron en cierto modo de estribo ideológico a la «política de la ciudad» que los socialistas dispusieron tras su ascenso al poder nacional, en el seno de equipos compuestos mayoritariamente por tránsfugas de esa «segunda izquierda» («Desarrollo social de los barrios, Mission Banlieue 89, Contrats de ville ${ }^{5}$ ).

Pero la izquierda política no fue la única en evolucionar en ese sentido: las políticas públicas francesas de los setenta observan una sustitución de las políticas urbanas por las «políticas del marco de vida». Durante esta década los sucesivos gobiernos de derecha bajo la presidencia de Valéry Giscard D'Estaing optaron por apoyarse progresivamente en la «participación de los ciudadanos», recuperar un urbanismo cualitativo, de «dimensión humana», mejorar el «marco de vida» y la calidad arquitectónica, reducir las escalas del urbanismo. Estas ideas rompían con el periodo precedente del gaullismo y rescataban los lemas de la izquierda y del movimiento social.

Durante este periodo se disponen los primeros programas «hábitat y vida social» $(\mathrm{HVS})^{6}$, los primeros dispositivos de la «política de la ciudad» que, sin embargo, no verían la luz sino en la década posterior. Hay que recordar que ciertos promotores de la «segunda izquierda» intervinieron en la disposición de esos procedimientos, pretendiendo «resocializar» los grandes conjuntos habitacionales mediante la «animación» y la «participación».

\section{SOCIOLOGÍA URBANA Y EVOLUCIÓN DE LAS POLÍTICAS URBANAS EN FRANCIA: EL «LUGAR», LA PARTICIPACIÓN Y EL ESPACIALISMO}

La evolución de la sociología urbana francesa acompaña al cambio que tiene lugar en las políticas públicas urbanas, proceso que podría designarse como el paso de la era de la «planificación» a la era del «proyecto» (Gaudin 1993). En los años 1970-1980 esa transición se caracteriza por la reducción de las escalas de la acción pública (de la ciudad al barrio), la apertura creciente a la financiación privada, la multiplicación de los actores implicados en los procesos de ordenación urbana (cuya «participación» habitante no constituye sino un ejemplo, y no el mejor vista su efectividad), y de forma más general, se caracteriza por

5 Se trata de los primeros dispositivos de la «política de la ciudad» desplegados a principios de los años 1980 por el gobierno socialista.

6 «Habitat et vie sociale», primeras formas contractuales entre el Estado francés y las municipalidades, que también prefiguran la «política de la ciudad» (como se ha especificado, la política aplicada en las periferias donde se levantan los grandes conjuntos) que se institucionalizará en los años 1980-1990.

EMPIRIA. Revista de Metodología de Ciencias Sociales. N. ${ }^{2}$ 27, enero-abril, 2014, pp. 121-136. ISSN: 1139-5737 DOI 10.5944/empiria.27.10865 
un marco menos voluntarista y dogmático y más «pragmático»: un urbanismo «de pensamiento débil» como lo calificaría más tarde el sociólogo Yves Chalas (2000, 2004).

Como hemos visto, en las décadas de 1960-1970 en Francia emerge y se desarrolla la noción de «marco de vida» aportada por las capas medias urbanas. Muy pronto, los partidos políticos (PSU, PCF, PS) crearon comisiones ad hoc, orientándose progresivamente, desde el punto de vista electoral, hacia dichas capas sociales.

Con esta noción de marco de vida cambia la escala de concepción política de la ciudad. Se enfatiza menos la «sociedad» que la «vida cotidiana» y globalmente supone una recentralización en el individuo en sociedad (y en el espacio). $\mathrm{Y}$ esto se realiza paralelamente al fomento de la escala local como ámbito pertinente de la gobernanza, de la comprensión de la ciudad y de la acción social y espacial (Genestier 1999). La escala local, la del barrio o del vecindario, es además susceptible de ser movilizada con comodidad en las reivindicaciones y en la contestación urbana.

También la sociología urbana y a fortiori la sociología del territorio ven cómo sus reflexiones y formas de aprehender la ciudad cambian de escala: de la ciudad en su globalidad y de los grandes esquemas de explicación y análisis de la urbanización, de la segregación y de la planificación, las investigaciones se reorientan durante los años 1980 hacia el barrio, el vecindario, la manzana, el inmueble, el espacio público (las sociabilidades, las interacciones...) y esto con la restitución de los trabajos de la Escuela de Chicago, traducidos al francés a finales de 1970 (Graffmeyer y Joseph 1979), y el desarrollo de la antropología urbana en Francia.

El giro localista está a la vez presente en las políticas urbanas — con el fin del llamado periodo de «planificación»— y en el seno de la sociología urbana francesa - con el declive del marxismo urbano y el auge de la antropología urbana. Los sociólogos ya no ambicionan discernir la urbanización o el fenómeno urbano en su globalidad, ni siquiera la ciudad en su conjunto: se inclinan hacia un nivel «micro». La «modestia» de la que habla Yves Chalas para calificar la acción pública y el «urbanismo de pensamiento débil» contemporáneos (tras el «urbanismo de pensamiento fuerte» de los Treinta gloriosos) podría aplicarse a la sociología urbana de los años 1980 y, así pues, llevarnos a hablar de una «sociología de pensamiento débil» asentada sobre la «incertidumbre» y el «pragmatismo», a diferencia de la «sociología de pensamiento fuerte» precedente, basada sobre la «verdad» y una «visión globalizadora» (Chalas 2000). Obrando así, la sociología de pensamiento débil franqueaba el análisis marxista en términos de clase, sin vocación de criticar la realidad socio-espacial ni de cambiarla en absoluto. La voluntad «utopista»o «utopiana» (Busquet 2004) de la sociología urbana, la de cambiar el mundo, desaparecía para dejar paso a una visión desencantada o despolitizada del orden establecido (Garnier 2008).

El corolario de este cambio de escala fue, con más claridad desde mitad de los años 1970, el auge de la idea del urbanismo participativo, de participación «ciudadana» (en sentido lato) en las decisiones y la ordenación, dentro de la ac-

EMPIRIA. Revista de Metodología de Ciencias Sociales. N. o 27, enero-abril, 2014, pp. 121-136. ISSN: 1139-5737 DOI 10.5944/empiria.27.10865 
ción pública. La idea de un urbanismo participativo fue igualmente - como se ha dicho- objeto de reivindicación de las capas medias urbanas y un eslogan de los sucesivos gobiernos desde la llegada al poder nacional de Giscard D'Estaing en 1974.

La participación en la planificación, avivada en los años 1960 por Lefebvre y Ledrut, parecía inconcebible cuando se trataba de concebir globalmente de la ciudad. En el plano local - a escala de la ordenación de proximidad de barrio- parecía sin embargo viable. En Francia, las nociones de «democracia de proximidad» o de «democracia local» (Bacqué 2002) para calificar la participación, opuestas a esa democracia representativa que supuestamente colmaría los límites, resultaban reveladoras: lo local debía ser comprendido no sólo como una escala política válida y pertinente (tanto para la toma de decisiones como para el movimiento social), sino también un entidad espacial pertinente desde el punto de vista de la reflexión y de la práctica urbanísticas. No sería cuestión de dar poder al ciudadano - ni de una autogestión ni siquiera en su modalidad «urbana» ${ }^{7}$ como era reclamada por los pensadores radicales y el movimiento social de los años 1960-1970 - sino de proporcionarle cautamente una senda de compromiso y concurso en los asuntos de su «vecindario» (o sea, «locales»), sobre objetos o problemáticas por otra parte impuestas y elegidas por quienes tienen potestad decisoria.

Por último, para combatir las segregaciones socio-espaciales, cada vez menos percibidas como emanación directa de las políticas públicas y de poblamiento, o como resultado de las estrategias de las unidades familiares y de elecciones individuales, el acento se pone igualmente en la «mezcla social», y eso tanto en las políticas de la ciudad como entre los sociólogos. Es el caso particular de los grandes polígonos de viviendas: cualquiera que fuere la interpretación dada, se trataría de luchar contra su relegación progresiva, denunciada desde los años 1960 por la sociología urbana marxista y los medios de comunicación; incluso se trataría de «romper los guetos». ${ }^{8}$

La cuestión de la escala del proyecto y de la comprensión de la ciudad, la de lo local (del «lugar» o del «barrio»), acarrea a la vuelta de los años 1970-1980 la idea de participación, y acompaña al paso de una forma a otra de acción pública, y de una sociología a otra, del «pensamiento fuerte» al «pensamiento débil», del «dogmatismo» al «pragmatismo», incluso de la utopía a la realidad de la evolución de los espacios urbanos.

El afianzamiento de la escala local como marco de intervención y de comprensión de lo urbano y de lo social, que escolta el fin del denominado periodo de «planificación», y la idea del urbanismo participativo que le es corolario, son

7 Esta noción, desarrollada por Henri Lefebvre, las luchas urbanas, la CFDT, el PSU y otros, remite a la toma en consideración por los habitantes y usuarios de las decisiones en materia urbanística y en la gestión urbana. Constituye una autogestión económica y política imposible a buen seguro en los modos de producción y gobernanza actual, siendo precisa una autogestión generalizada (a nivel del poder y de la empresa).

8 Esta cuestión de la mezcolanza social ya estaba presente durante la concepción de los grandes conjuntos: se trataba de mezclar las capas medias y las populares.

EMPIRIA. Revista de Metodología de Ciencias Sociales. N. . 27, enero-abril, 2014, pp. 121-136. ISSN: 1139-5737 DOI 10.5944/empiria.27.10865 
a la vez guiadas por la «segunda izquierda», el movimiento social, las capas medias urbanas y los sociólogos de lo urbano. Mediante esos actores y ese nuevo tópico del «marco de vida» se realiza una «urbanización» del discurso y de las posiciones de la izquierda política en Francia inmediatamente después de los Treinta gloriosos. La emergencia de la temática del «marco de vida» llevada por las capas medias, la nueva derecha, la «segunda izquierda» y los sociólogos de los años setenta marca, pues, tanto una politización de lo local como una espacialización de lo político.

Pero de esta manera asistimos igualmente a una despolitización relativa en la concepción del fenómeno urbano: si lo local se politiza (si deviene objeto de las políticas públicas y escala pertinente de la democracia soñada), la ciudad y lo urbano son decrecientemente percibidas como terreno de las segregaciones, de las desigualdades y de las luchas, por simbólicas que fueren. Se tratará en adelante, para las políticas públicas, de «administrar» o de «atender» a los barrios de la exclusión, los «barrios del exilio» y de la «relegación» (Dubet, Lapeyronnie, 1992); y para la sociología urbana se tratará de describir la «anomia», la «exclusión», incluso la tendencia de los barrios a devenir en «guetos», desarrollando de paso categorías recogidas después por la acción pública (Tissot 2007).

La nueva visión de la ciudad también comporta en sí misma las soluciones a la situación, que en lo sucesivo se plantean más en términos cualitativos que cuantitativos. Por lo demás sigue siendo una visión teñida de utopía, como las de antaño: en los años 1960, para los críticos del urbanismo y hasta entre los sociólogos, la alienación y los problemas sociales eran más evidentes en la ciudad y en tanto que derivaban igualmente del modo de producción del espacio era también en y por el espacio como deberían inscribirse los remedios. Pero la noción de marco de vida permite al espacio urbano adquirir un estatuto particular en el pensamiento político, reforzando su eventual papel en la resolución de los problemas sociales urbanos. El espacio urbano ya no es simplemente percibido como el soporte o terreno de las políticas públicas o de sus oposiciones sino que adquiere un estatuto cualitativo de primera importancia, social y políticamente, en términos de democracia y participación. Las soluciones a la anomia, a la exclusión y más generalmente a todos los problemas sociales urbanos habrán de ser encontradas en la escala o nivel local, con abundancia de «participación» - lo que no excluye la acción sobre el espacio para actuar sobre lo social, muy al contrario. Esta actuación sería incluso factible en el seno de la acción pública «de pensamiento débil». La ideología espacialista (Garnier, Goldschmidt, 1978; Chalas 1997; Garnier, 2002; Busquet 2007, 2009), es decir, la idea según la cual existiría un determinismo causal entre el espacio construido y los hechos sociales, no desaparece con la crisis y tras el urbanismo voluntarista «de pensamiento fuerte», simplemente cambia de escala de aplicación y de contenido, remitiéndose a la calidad de vida y a la vida cotidiana (Busquet 2007).

En suma, si de alguna manera la sociología urbana francesa ha tenido influencia sobre las políticas públicas ha sido a través de una nebulosa de actores y de sus respectivas ideas sobre las relaciones entre espacios y grupos sociales. Esta nebulosa aglutina a los representantes de la «segunda izquierda» francesa

EMPIRIA. Revista de Metodología de Ciencias Sociales. N. ${ }^{\circ}$ 27, enero-abril, 2014, pp. 121-136. ISSN: 1139-5737 DOI 10.5944/empiria.27.10865 
con los sociólogos urbanos cuyos objetos se reorientan hacia la escala local. Se podría advertir una evolución concomitante al ver el impacto sobre la acción pública, sin poder determinar, no obstante, quién de entre los paladines de la «segunda izquierda» o de la sociología urbana ha tenido más influencia, incluso cuando los préstamos de los primeros a los segundos (el derecho a la ciudad, principalmente) han sido demostrados en otra parte (Busquets 2007). A cambio, claro, las políticas públicas y su evolución han proporcionado evidentemente nuevos paradigmas a los sociólogos.

Sea como fuere, se pueden observar continuidades entre la oposición a las políticas urbanas gaullista en los años 1960-1970 y la política de la ciudad de los años 1980: continuidad de ideas, de temática (la mezcla social, la participación), de actores, pero también continuidad en la comprensión de las relaciones entre el espacio y las sociedades urbanas (el espacialismo), bien se manifiesten en forma de eslóganes, bien de prácticas de gestión, bien en la reforma de los territorios urbanos.

\section{5. ¿Y AHORA? LA SOCIOLOGÍA Y LA ACCIÓN PÚBLICA}

Me gustaría concluir con algunas perspectivas en forma de hipótesis o de cuestionamientos concernientes a los impactos y a la posterior evolución de esas relaciones entre la sociología urbana y la acción pública territorializada: si la sociología urbana francesa contempla vínculos privilegiados con la acción pública, provocando en cierto modo la evolución ideológica de una y otra, podemos plantear la cuestión del devenir de dichas relaciones a partir de los años 1980.

Durante esa década, el reflujo del marxismo en las ciencias sociales podría hacernos pensar en principio en una contracción de la crítica en la sociología urbana, ya que durante más de un decenio se había basado en dicho paradigma para contradecir el urbanismo estatal o privado, criticando sus efectos sociales. Desde los años 1960, esta sociología había elegido la «modernidad» urbana para criticarla desde diferentes ángulos, tratando de modificar la acción pública con el apoyo de los movimientos sociales.

Hoy día se puede constatar que esta sociología urbana ha perdido la iniciativa9 ${ }^{9}$ : sus temas le son progresivamente impuestos, resulta cada vez más dependiente de una demanda tematizada (siendo el «desarrollo urbano sostenible» o la «ciudad sostenible» las últimas ocurrencias). Mientras que los sociólogos de los años 1960 promovían los lemas del movimiento social en los medios políticos, criticando las políticas urbanas realizadas con subsidios del Estado, hoy se puede observar una tendencia al acantonamiento del sociólogo urbano en el rol de evaluador (como, por ejemplo, en el caso de «la política de la ciudad, cuyas transformaciones se basan aparentemente en estimaciones negativas o positivas» ${ }^{10}$ )

\footnotetext{
9 Si alguna vez la tuvo. Es lo que siempre reivindicaba y ya no reivindica.

10 Habría mucho que decir por lo demás sobre esta cuestión de la evaluación, que podría permitir canalizar la crítica —incluso la investigación — anclada a los «marcos» de la acción pública.
}

EMPIRIA. Revista de Metodología de Ciencias Sociales. N. . 27, enero-abril, 2014, pp. 121-136. ISSN: 1139-5737 DOI 10.5944/empiria.27.10865 
o, en el extremo, el papel de cuasi-prospectivista (en el caso de la ciudad o el desarrollo sostenibles, temas que capitalizan cada vez más investigaciones). Ciertamente, el papel de consultor o de consejero áulico del sociólogo urbano no es reciente: ya estaba vigente, como los gabinetes de estudio, en los años 1960 y fue cuestionado durante el coloquio de Royaumont de 1968.

Pero en la actualidad el aspecto crítico de la sociología queda enmarcado por sus propios objetos de investigación. El sociólogo urbano siempre ha mantenido en su ánimo, quizá por obediencia disciplinar, una distancia crítica respecto a sus objetos de estudio, que le lleva a todo tipo de bricolajes intelectuales (cuestionar la sostenibilidad en los estudios que versan sobre el desarrollo sostenible, criticar los efecto socio-espaciales de las «políticas de la ciudad» o de mezcla social residencial de las que es parte integrante en calidad de «experto», o incluso el sesgo de la «investigación-acción», heredera de la sociología urbana en Francia).

Si la crítica social global en el sentido que se le daba en los años 1960-1970 ha desaparecido (¡la reorientación sobre los objetos locales obliga!), el sociólogo urbano se pretende siempre un sujeto comprometido.

La sociología urbana cuestiona, en la medida de lo posible, su propia posición en la ciudad -incluso mucho menos que su papel, cada vez más encuadrado o estipulado- sin pretender o querer actuar más sobre lo social, en nombre de una neutralidad o de una voluntad de objetivación recurrente (Garnier 2008), a menudo dejando hoy la crítica de la acción pública a otros. Y si el espacio es siempre un producto y un objetivo políticos (Lefebvre 1973), el sociólogo urbano lo es cada vez menos. La cuestión de las relaciones entre la ciencia social y el poder, si pueden analizarse mediante la comprensión de las políticas públicas, podría examinarse también en términos de posturas individuales y colectivas.

\section{BIBLIOGRAFÍA}

AMIOT, M. (1986) : Contre l'Etat, les sociologues. Eléments pour une histoire de la sociologie urbaine en France (1900-1980), Paris, éditions de l'EHESS.

BACHMANN, Ch., LE GUENNEC, N. (1995) : Violences urbaines : ascension et chute des classes moyennes à travers cinquante ans de politique de la ville, Paris, A. Michel.

BACQUÉ, M.-H. (2002) : «Démocratie participative-démocratie de proximité^», in : Conseils de quartier/mode d'emploi. ADELS.

BACQUÉ, M.-H., VERMEERSCH, S. (2007) : Changer la vie : les couches moyennes et l'héritage de mai 68, Paris, Editions de l'atelier.

BIDOU, C. (1984) : Les Aventuriers du quotidien, Paris, PUF.

BUSQUET, G. (2004), « Henri Lefebvre, L'Internationale situationniste et la revue Utopie $^{\wedge}$ », Urbanisme, n. ${ }^{\circ} 336$ (dossier «Utopies»), mai — juin 2004, pp. 54-57.

BUSQUET, G. (2007), Idéologie urbaine et pensée politique dans la France de la période 1958-1981, thèse de doctorat en urbanisme et aménagement, Institut d'urbanisme de Paris, Université Créteil-Val de Marne. 
BUSQUET, G. (2007b) : «L'idéologie et l'espace urbain dans les années 60-70 : le cas du discours du Parti socialiste unifié^^^, in : VIALA Laurent, VILLEPONTOUX Stéphane (sous la dir. de), 2007, Imaginaire, territoires, sociétés. Contribution à un déploiement transdisciplinaire de la géographie social, MTE - CNRS, Montpellier, Publications de l'Université Paul-Valéry-Montpellier III.

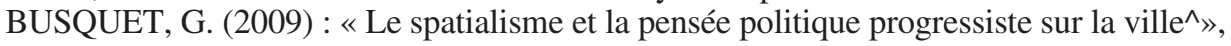
in BAUDIN, G., BONNIN, Ph., Faire territoire aujourd'hui, Paris, Editions Recherches, 281-299

CASTELLS, M. (1972) : La Question urbaine, Paris, F. Maspero, 1975.

CASTELLS, M. (1968) : «Y'a-t-il une sociologie urbaine ?^», Sociologie du travail, Paris, Seuil, 72-90.

CASTELLS, M., GODARD, F. (1974): Monopolville. L'entreprise, l'Etat, l'urbain, Paris-La Haye, Mouton.

Centre de Sociologie Urbaine (1981) : L’Activité scientifique du Centre de Sociologie Urbaine (1978-1981), Paris, CSU.

CHALAS, Y. (1997) : Urbanité et périphérie. Connaissance et Reconnaissance des territoires contemporains, Paris, ministère du Logement, Plan Construction et Architecture.

CHALAS, Y. (2000) : L'Invention de la ville, Paris, Anthropos.

CHALAS, Y. (2004) : «La gouvernance ouverte au débat public ou la pensée faible comme refondation de l'action publique ${ }^{\wedge}$, in : CHALAS, Y. (sous la dir. de), L'Imaginaire aménageur en mutation. Cadres et référents nouveaux de la pensée et de l'action urbanistiques. Contribution au débat, Paris, L'Harmattan.

CHAMBOREDON, J.-C., LEMAIRE, M. (1970) : «Proximité spatiale et distance sociale : les grands ensembles et leur peuplement», Revue française de sociologie, ${ }^{\circ}{ }^{\circ}$ XI-1, Paris.

CHOMBART DE LAUWE, P.-H. (1963) : Des hommes et des villes, Paris, Payot, 1973.

COING, H., TOPALOV, Ch. (1995) : «Crise, urgence et mémoire : où sont les vraies ruptures $?^{\wedge} »$, in : ASCHER, F. (coord.), Le Logement en question, Paris, Editions de l'Aube, 261-290.

DAGNAUD, M., MEHL, D. (1981) : «Des contestataires comme il faut ${ }^{\wedge}$, Autrement, n. ${ }^{\circ} 29$.

DUBET, F., LAPEYRONNIE, D. (1992) : Les Quartiers d'exil, Paris, Seuil.

Fondation Royaumont pour le progrès des sciences de l'homme (1970) : Sociologie et urbanisme, colloque des 1, 2 et 3 mai 1968, compte-rendu rédigé par Jean-Paul Trystram, Paris, Editions de l'Epi.

GARNIER, J.-P., GOLDSCHMIDT, D. (1978) : La Comédie urbaine ou la cité sans classe, Paris, F. Maspero.

GARNIER, J.-P. (2002) : «Quelle politique de la ville ?^^, tribune de Politis, 14 février 2002.

GARNIER, J.-P. (2008) : «La Volonté de non-savoir^^, Villes et résistances sociales, Agone, n. ${ }^{\circ}$ 38-39, Marseille, 49-70, republié dans Une violence éminemment contemporaine. Essais sur la ville, la petite bourgeoisie intellectuelle \& l'effacement des classes populaires, Marseille, Contre-feux, Agone.

GENESTIER, Ph. (1999) : «Le sortilège du quartier : quand le lieu est censé faire lien », Les Annales de la recherche urbaine, $\mathrm{n} .{ }^{\circ} 82$.

GRAFMEYER, Y., JOSEPH, I. (éd.) (1979) : L'Ecole de Chicago. Naissance de l'écologie urbaine, Paris, Aubier, 1998. 
GAUDIN, J.-P. (1993) : Les Nouvelles politiques urbaines, Paris, PUF, Coll. « Que sais-je ?», n. ${ }^{\circ} 2839$.

LASSAVE, P. (1997) : Les Sociologues et la recherche urbaine dans la France contemporaine, Toulouse, Presses Universitaires du Mirail.

LEFEBVRE, H. (1968) : Le Droit à la ville, Paris, Seuil, 1974

LEFEBVRE, H. (1973) : Le Droit à la ville II : Espace et politique, Paris, Anthropos, 2000.

LEFEBVRE, H. (1970) : La Révolution urbaine, Paris, Gallimard.

LOJKINE, J. (1977) : Le Marxisme, l'Etat et la question urbaine, Paris, PUF.

MAURIN, E. (2004) : Le ghetto français. Enquête sur le séparatisme social, Paris, Seuil. TISSOT, S. (2007) : L'Etat et les quartiers. Genèse d'une catégorie de l'action publique, Paris, Seuil.

TOURAINE, A. (1978) : La Voix et le regard. Sociologie des mouvements sociaux, Paris, Le Seuil, 1993. 